\title{
BOUNDEDNESS OF CERTAIN AUTOMORPHISM GROUPS OF AN OPEN MANIFOLD
}

\author{
TOMASZ RYBICKI
}

\begin{abstract}
It is shown that certain diffeomorphism or homeomorphism groups with no restriction on support of an open manifold (being the interior of a compact manifold) are bounded. It follows that these groups are uniformly perfect. In order to characterize the boundedness several conditions on automorphism groups of an open manifold are introduced. In particular, it is shown that the commutator length diameter of the automorphism group $\mathcal{D}(M)$ of a portable manifold $M$ is estimated by 4 .
\end{abstract}

\section{InTRODUCTION}

Let us recall that a group is called bounded if it is bounded with respect to any bi-invariant metric. The purpose of this paper is to show that some diffeomorphism or homeomorphism groups with not necessarily compact support of an open manifold are bounded. We will formulate some conditions which ensure the boundedness of such groups. Throughout, to avoid complications in terminology we will refer to the homeomorphisms as diffeomorphisms (of class $C^{0}$ ).

In the sequel we will deal with a manifold $M$ being the interior of a compact manifold $\bar{M}$. We adopt the following notation similar to that in [16]. Let $\partial_{i}, i=1, \ldots, k$, be the family of all connected components of the boundary $\partial$ of $\bar{M}$. Let $K=\{1, \ldots, k\}$. For any $J \subset K$ and $r=0,1, \ldots, \infty$ the symbol $\operatorname{Diff}_{J}^{r}(M)$ will stand for the totality of $C^{r}$-diffeomorphisms which are equal to the identity on a neighborhood of $\partial_{J}:=\bigcup_{i \in J} \partial_{i}$. Then $\operatorname{Diff}^{r}(M)=$ $\operatorname{Diff}_{\emptyset}^{r}(M)$ and $\operatorname{Diff}_{c}^{r}(M)=\operatorname{Diff}_{K}^{r}(M)$, the group of compactly supported $C^{r}$ diffeomorphisms of $M$. Next, $\mathcal{D}_{J}^{r}(M)$ denotes the group of all elements of $\operatorname{Diff}_{J}^{r}(M)$ that can be joined with the identity by an isotopy in $\operatorname{Diff}_{J}^{r}(M)$. In particular, $\mathcal{D}^{r}(M)=\mathcal{D}_{\emptyset}^{r}(M)$ (resp. $\left.\mathcal{D}_{c}^{r}(M)=\mathcal{D}_{K}^{r}(M)\right)$ is the (resp. compactly supported) identity component of the group of all $C^{r}$-diffeomorphisms of $M$.

Date: revised; June 7, 2010.

1991 Mathematics Subject Classification. 22E65, 57R50, 57S05.

Key words and phrases. Open manifold, bounded group, conjugation-invariant norm, group of diffeomorphisms, commutator length, perfectness, uniform perfectness.

Partially supported by the Polish Ministry of Science and Higher Education and the AGH grant n. 11.420.04. 
The problem of the boundedness of a group of diffeomorphisms is closely related to its uniform perfectness (c.f. [5], Propositions 1.3 and 1.4). Recall that a group $G$ is called perfect if it is equal to its own commutator subgroup $[G, G]$. Next $G$ is said to be uniformly perfect if $G$ is perfect and there exists a positive integer $N$ such that any element of $G$ can be expressed as a product of at most $N$ commutators of elements of $G$. For $g \in[G, G]$ the least $N$ such that $g$ is a product of $N$ commutators is called the commutator length of $g$ and is denoted by $\mathrm{cl}_{G}(g)$.

If $\partial \bar{M}=\emptyset$ then it is well-known that $\mathcal{D}^{r}(M)$ is a simple group, where $r=0,1, \ldots, \infty$, except possibly $r=\operatorname{dim}(M)+1$ (c.f. for $r=0$ [14] with [7], and for $r \geq 1$ [21] and [15]). Another basic theorem was proved by D. McDuff in [16].

Theorem 1.1. [16] Suppose that $\partial \bar{M} \neq \emptyset$. The groups $\mathcal{D}_{J}^{r}(M)$ are perfect unless $J=K$ and $r=\operatorname{dim}(M)+1$. In particular, $\mathcal{D}^{r}(M)$ is a perfect group.

In the sequel we will always assume that $\partial \bar{M} \neq \emptyset$.

In 2009 P. Schweitzer reconstructed in 20] his own proof of the fact that the quotient $\mathcal{D}^{r}(M) / \operatorname{Diff}_{\{i\}}^{r}(M) \cap \mathcal{D}^{r}(M), i \in K$, is a simple group. This theorem, which is a "hardest work" ([16]) in the proof of Theorem 1.1, had been proved independently by W. Ling, Schweitzer and McDuff more than thirty years ago, but the proof has never been published.

Recently, basic results concerning the uniform perfectness and the boundedness of diffeomorphism groups of many manifolds have been proved by D. Burago, S. Ivanov and L. Polterovich in [5] and by T. Tsuboi in [23]. In contrast to the problem of perfectness and simplicity, these results depend essentially on the topology of the underlying manifold. These results generalize older ones, e.g. [2].

Note that the problem of the uniform perfectness and the boundedness is still valid for some nontransitive diffeomorphism groups which are perfect but non-simple, e.g. for the diffeomorphism group of manifold with boundary ([18]), or of a foliated manifold ([17], [22], [12]). For the problem of perfectness and uniform perfectness in the relative case of $\operatorname{Diff}(M, N)$, where $N$ is a proper submanifold of $M$, see [19] and [1].

In section 2 we will show the equivalence of many conditions describing automorphism groups of an open manifold. In particular, as a consequence of these results we have

Theorem 1.2. Let $M$ be a manifold of class $C^{r}, r=0,1, \ldots, \infty$, as above and let $M$ be portable or, more generally, let $M$ satisfy the ( $\sqcup)$-property (c.f. Def.2.14). Let $J \subset K$ and $r \neq \operatorname{dim}(M)+1$. Then the group $\mathcal{D}_{J}^{r}(M)$ is bounded. In particular, $\mathcal{D}^{r}(M)$ is bounded. 
Notice that the class of portable manifolds includes the euclidean spaces $\mathbb{R}^{n}$, the manifolds of the form $M \times \mathbb{R}^{n}$, and the three-dimensional handlebodies.

Corollary 1.3. Under the above assumptions, the group $\mathcal{D}_{J}^{r}(M)$ is uniformly perfect. The commutator length diameter of $\mathcal{D}_{J}^{r}(M)$ is $\leq 4$.

The first statement is an immediate consequence of Theorem 1.2, the second will be proved in section 2 . In section 2 we also obtain another estimation on the diameter of $\operatorname{cl}_{\mathcal{D}_{J}^{r}(M)}$, c.f. (2.2), for a much wider class of automorphism groups.

In our investigations a special role is played by the properties that an automorphism group is "factorizable" or that it is "determined on compact subsets" (Definitions 2.1 and 2.5(2)). The significance of these properties is illustrated by examples in section 3 .

Acknowledgments. A correspondence with Paul Schweitzer and his recent paper [20] were helpful when I was preparing my paper. I would like to thank him for his kindness. I also express my deep gratitude to the referee for pointing out some unclear or wrong statements, especially in a previous version of Def. 2.1, and for other valuable remarks.

\section{BOUNDEDNESS AND UNIFORM PERFECTNESS OF CERTAIN AUTOMORPHISM GROUPS}

The notion of the conjugation-invariant norm is an indispensable tool in studies of the boundedness of groups. Let $G$ be a group. A conjugationinvariant norm on $G$ is a function $\nu: G \rightarrow[0, \infty)$ which satisfies the following conditions. For any $g, h \in G$

(1) $\nu(g)>0$ if and only if $g \neq e$;

(2) $\nu\left(g^{-1}\right)=\nu(g)$;

(3) $\nu(g h) \leq \nu(g)+\nu(h)$;

(4) $\nu\left(h g h^{-1}\right)=\nu(g)$.

It is easily seen that $G$ is bounded if and only if any conjugation-invariant norm on $G$ is bounded.

Suppose that $G$ is perfect. Then the commutator length $\mathrm{cl}_{G}$ is a conjugationinvariant norm on $G$.

Recall that $M$ is the interior of a compact, connected manifold $\bar{M}$ of class $C^{r}$, where $r=0,1, \ldots, \infty$, with non-empty boundary $\partial=\partial_{1} \cup \ldots \cup \partial_{k}$. By a product (or collar) neighborhood of $\partial$ we mean a closed subset $P=\bigcup_{i=1}^{k} P^{(i)}$ of $M$, where $P^{(i)}=\partial_{i} \times[0,1)$, so that $P=\partial \times[0,1)$. Here $\partial \times[0,1]$ is embedded in $\bar{M}$, and $\partial \times\{1\}$ is identified with $\partial$. In particular, $P^{(i)}$ are pairwise disjoint.

A translation system on the product manifold $N \times[0, \infty)$ (c.f. [13], p.168) is a family $\left\{P_{j}\right\}_{j=1}^{\infty}$ of closed product neighborhoods of $N \times\{\infty\}$ such that $P_{j+1} \subset \operatorname{Int} P_{j}$ and $\bigcap_{j=1}^{\infty} P_{j}=\emptyset$. A detailed description of the role played by 
translation systems on product manifolds $N \times \mathbb{R}$ was given in Ling's paper [13. By a translation system of $M$ we understand a translation system on a product neighborhood of $\partial_{i}, P^{(i)}=\partial_{i} \times[0,1)$, where $i \in K$. By a ball we mean an open ball with its closure compact and contained in a chart domain.

Let $G$ be a subgroup of $\operatorname{Diff}^{r}(M)$. For a subset $U \subset M$ denote by $G(U)$ the subgroup of all elements of $G$ which can be joined with the identity by an isotopy in $G$ compactly supported in $U$. Recall that the support of a diffeomorphism $f$ on $M$, denoted by $\operatorname{supp}(f)$, is the closure of the set $\{x \in M$ : $f(x) \neq x\}$, and the support of an isotopy $\left\{f_{t}\right\}_{t \in[0,1]}$ is defined by $\operatorname{supp}\left(\left\{f_{t}\right\}\right):=$ $\bigcup_{t} \operatorname{supp}\left(f_{t}\right)$.

We say that $g \in G$ meets the $i$-th end of $M(i \in K)$ if for any neighborhood $U$ of $\partial_{i}$ we have $U \cap \operatorname{supp}(g) \neq \emptyset$, i.e. if $g$ does not stabilize near $\partial_{i}$. Next, we say that $G$ meets the $i$-th end of $M$ if there is $g \in G$ which does so. Denote $J_{G}=\{i \in K: G$ meets the $i$-th end of $M\}$, and $\partial_{G}=\bigcup_{i \in J_{G}} \partial_{i}$.

Definition 2.1. $G$ is called factorizable if for any $g \in G$ there are a family of balls $\left\{B_{\alpha}\right\}_{\alpha \in A}$, a product neighborhood $P=\partial \times[0,1)$, and a family of diffeomorphisms $g_{j} \in G$ for $j=0,1, \ldots, N$ such that:

(1) $g=g_{0} g_{1} \cdots g_{N}$ with $g_{0} \in G(P)$ and $g_{j} \in G\left(B_{\alpha(j)}\right), j=1, \ldots, N$.

Moreover, for any product neighborhood $P$ and for any $g \in G(P)$ there is a sequence of reals from $(0,1)$ tending to 1

$$
0<a_{1}<\bar{a}_{1}<\bar{b}_{1}<b_{1}<a_{2}<\ldots<a_{n}<\bar{a}_{n}<\bar{b}_{n}<b_{n}<\ldots<1
$$

and $h \in G(P)$ such that

(2) $h=g$ on $\bigcup_{n=1}^{\infty} \partial \times\left[\bar{a}_{n}, \bar{b}_{n}\right]$;

(3) $h=$ id on $P^{(i)}, i \in K$, whenever $g=$ id on $P^{(i)}$.

Put $D_{n}:=\partial \times\left(a_{n}, b_{n}\right)$ and $D:=\bigcup_{n=1}^{\infty} D_{n}$. Then we also assume that:

(4) $\operatorname{supp}(h) \subset D$;

(5) for the resulting decomposition $h=h_{1} h_{2} \ldots$ with respect to $D=$ $\bigcup_{n=1}^{\infty} D_{n}$ we have $h_{n} \in G\left(D_{n}\right)$ for all $n$.

Remark 2.2. The reason for formulating Def. 2.1 is the absence of isotopy extension theorems or fragmentation theorems for automorphism groups of some geometric structures. Roughly speaking, $G$ satisfies Def. 2.1 if all its elements can be joined with id by an isotopy in $G$ and appropriate versions of the above mentioned theorems are available.

Let $G$ be factorizable. Then for any $g \in G$ there are a family of balls $\left\{B_{\alpha}\right\}$, a product neighborhood $P$ of $\partial$, and a decomposition

$$
g=g_{01} \ldots g_{0 k} g_{1} \ldots g_{N},
$$

where $g_{0 i} \in G\left(P^{(i)}\right), i=1, \ldots, k$, and $g_{j} \in G\left(B_{\alpha_{j}}\right), j=1, \ldots, N$. For $J \subset K$ we define $G_{J}$ as the totality of $g \in G$ such that there is a decomposition (2.1) 
with $g_{i 0}=$ id for all $i \in J$. We also put $G_{c}=G_{K}$. Then it is easily checked the following

Proposition 2.3. If $G$ is factorizable then so are $G_{J}$. Moreover, if $G$ is factorizable then $G$ (resp. $\left.G_{J}\right)$ are contained in $\mathcal{D}^{r}(M)\left(\right.$ resp. $\left.\mathcal{D}_{J}^{r}(M)\right)$. We also have $J_{G_{J}} \subset K \backslash J$.

Example 2.4. The group $\operatorname{Diff}^{r}\left(\mathbb{R}^{n}\right)$ does not satisfy Def.2.1. The reason is that in this case any $f \in \operatorname{Diff}^{r}\left(\mathbb{R}^{n}\right)$ would be isotopic to id due to 2.1(1) which is not true. On the other hand, any $f \in \operatorname{Diff}_{c}^{r}\left(\mathbb{R}^{n}\right)$ is isotopic to the identity but the isotopy need not be compactly supported. It follows that $\operatorname{Diff}_{c}^{r}\left(\mathbb{R}^{n}\right)$ does not fulfil Def.2.1.(1). The exception is $r=0$, when the Alexander trick is in use and any compactly supported homeomorphism on $\mathbb{R}^{n}$ is isotopic to id by a compactly supported isotopy. It follows that $\operatorname{Diff}_{c}^{0}\left(\mathbb{R}^{n}\right)$ is factorizable in view of [7].

Let $C=\mathbb{R} \times \mathbb{S}^{1}$ be the annulus. Then there is the twisting number epimorphism $\operatorname{Diff}_{c}^{r}(C) \rightarrow \mathbb{Z}$. It follows that $\operatorname{Diff}_{c}^{r}(C)$ is unbounded in view of Lemma 1.10 in [5]. On the other hand, $\operatorname{Diff}_{c}^{r}(C)$ is not factorizable. (This part of example was suggested by the referee.)

Definition 2.5. (1) $G$ is called locally perfect if there exists a covering by balls $\mathcal{B}$ of $M$ such that for any ball $B \in \mathcal{B}$ the subgroup $G(B)$ is perfect.

(2) $G$ is said to be determined on compact subsets if the following is satisfied. Let $f \in \mathcal{D}^{r}(M)$. If there are a sequence of relatively compact subsets $U_{1} \subset \bar{U}_{1} \subset U_{2} \subset \ldots \subset U_{n} \subset \bar{U}_{n} \subset U_{n+1} \subset \ldots$ with $\bigcup U_{n}=M$ and a sequence $\left\{g_{n}\right\}, n=1,2, \ldots$, of elements of $G$ such that $\left.f\right|_{U_{n}}=\left.g_{n}\right|_{U_{n}}$ for $n=1,2, \ldots$, then we have $f \in G$.

(3) We say that $G$ admits translation systems if for any $i \in J_{G}$ and for any sequence $\left\{\lambda_{n}\right\}, n=0,1, \ldots$, with $\lambda_{n} \in(0,1)$, tending increasingly to 1 , there exists a $C^{r}$-mapping $[0, \infty) \ni t \mapsto f_{t} \in G$ supported in the interior of $P^{(i)}$, with $f_{0}=\mathrm{id}, f_{j}=\left(f_{1}\right)^{j}$ for $j=2,3, \ldots$, and such that for the translation system $P_{n}=\partial_{i} \times\left[\lambda_{n}, 1\right)$ one has $f_{1}\left(P_{n}\right)=P_{n+1}$ for $n=0,1,2, \ldots$.

The following is a version of Isotopy Extension Theorem (c.f. [7], [10]).

Theorem 2.6. Let $f_{t}$ be an isotopy in $\mathcal{D}^{r}(M)$ and let $C \subset M$ be a compact set. Then for any open neighborhood $U$ of $\bigcup_{t \in[0,1]} f_{t}(C)$ there is an isotopy $g_{t}$ in $\mathcal{D}^{r}(M)$ such that $g_{t}=f_{t}$ on $C$ and $\operatorname{supp}\left(g_{t}\right) \subset U$.

Proposition 2.7. The groups $\mathcal{D}_{J}^{r}(M)(J \subset K)$ satisfy Definitions 2.1 and 2.5 with the possible exception for 2.5(1) in case $r=\operatorname{dim}(M)+1$. In particular, if $G=\mathcal{D}^{r}(M)$ then $G_{J}=\mathcal{D}_{J}^{r}(M)$. 
Proof. The proof will be written for $\mathcal{D}^{r}(M)$ (for $\mathcal{D}_{J}^{r}(M)$ is the same). For open $U \subset M$ let $\mathcal{D}^{r}(U)$ denote the group of all elements of $\mathcal{D}^{r}(M)$ that can be joined with the identity by an isotopy supported in $U$.

Def. 2.1: Let $g \in \mathcal{D}^{r}(M)$ and let $\tilde{g}_{t}$ be an isotopy joining $g$ with the identity. Take $\left\{B_{\alpha}\right\}$ and $P$ such that for $U=\bigcup_{\alpha \in A} B_{\alpha}$ we have $\overline{M \backslash P} \subset U$. Choose a compact subset $C=\partial \times[\lambda, \mu] \subset U$, where $0<\lambda<\mu<1$. Possibly enlarging $U$, by Theorem 2.6 there is an isotopy $f_{t}$ in $\mathcal{D}^{r}(U)$ such that $f_{t}=\tilde{g}_{t}$ on $C$. Put $h_{t}=f_{t}^{-1} \tilde{g}_{t}$. Then $\left.h_{t}\right|_{C}=$ id and $h_{t}=\tilde{g}_{t}$ off $U$. Set $h_{t}=\tilde{h}_{t} k_{t}$, where $\tilde{h}_{t} \in \mathcal{D}^{r}(\operatorname{Int}(P)), \operatorname{supp}\left(k_{t}\right) \subset U$, and $\operatorname{supp}\left(\tilde{h}_{t}\right) \cap \operatorname{supp}\left(k_{t}\right)=\emptyset$. It follows that $\bar{g}_{t}=\tilde{h}_{t}^{-1} \tilde{g}_{t}$ has support in $U$. Set $g_{0}=\tilde{h}_{1}$. By a fragmentation property for isotopies ([3], [7]) we get $\bar{g}_{1}=g_{1} \cdots g_{N}$ with $g_{j} \in \mathcal{D}^{r}\left(B_{\alpha(j)}\right)$ for $j=1, \ldots, N$. Clearly $g=g_{0} g_{1} \cdots g_{N}$, hence (1).

To show (2)-(5) we apply Theorem 2.6. This enables us to define recurrently $a_{n}<\bar{a}_{n}<\bar{b}_{n}<b_{n}$ and $\left.h\right|_{\left[a_{n}, b_{n}\right]}$ for $n=1,2, \ldots$ in such a way that the claim is fulfilled. In fact, let $g_{t}$ be an isotopy joining $g$ with the identity and supported in $\operatorname{Int}(P)$ and suppose we have defined $0<a_{1}<\ldots<b_{n-1}$. It suffices to take $b_{n-1}<a_{n}<\bar{a}_{n}<\bar{b}_{n}<b_{n}$ in such a way that $\partial \times\left[a_{n}, 1\right)$ is disjoint with $\bigcup_{t \in[0,1]} g_{t}^{-1}\left(\partial \times\left[0, b_{n-1}\right]\right)$ and use Theorem 2.6.

Def. 2.5: (1) It is a consequence of the fundamental results on the simplicity of diffeomorphism groups ([14], [21], [15], [7]). (2) is trivial.

(3) Let $\lambda_{n}>0$ be as above. Let $\tau:[0,1] \rightarrow \operatorname{Diff}^{\infty}([0,1))$ be an isotopy such that $\tau_{0}=$ id and $\tau_{1}\left(\lambda_{n}\right)=\lambda_{n+1}$ for $n=0,1,2, \ldots$ Next, let $\tau:[1,2] \rightarrow$ $\operatorname{Diff}^{\infty}([0,1))$ be an isotopy from $\tau_{1}$ to $\tau_{2}=\left(\tau_{1}\right)^{2}$. Continuing this procedure with $\tau_{j}=\left(\tau_{1}\right)^{j}$, let $\tau=\left.\bigcup_{j=0}^{\infty} \tau\right|_{[j, j+1]}:[0, \infty) \rightarrow \operatorname{Diff}^{\infty}([0,1))$, where $\tau$ is smoothed on neighborhoods of $j=1,2, \ldots$ if necessary. Set $f_{t}=\operatorname{id}_{P^{(i)}} \times \tau_{t}$.

Of course, if $g \in \operatorname{Diff}_{c}^{r}(C)$, where $C=\mathbb{R} \times \mathbb{S}^{1}$, is a diffeomorphism with nonzero twisting number, then $h$ determined by Def. 2.1, (2)-(5), is no longer compactly supported and does not extend to the boundary $\partial$.

The following fact shows that the statement " $g_{0} \in G(P)$ " in Def. 2.1(1) could be replaced by a weaker one $" \operatorname{supp}\left(g_{0}\right) \subset P$ ", provided $G$ fulfills Def. $2.5(3)$.

Proposition 2.8. Suppose that a group $G \subset \operatorname{Diff}^{r}(M)$ admits translation systems (Def. 2.5(3)). If $g \in G$ with $\operatorname{supp}(g) \subset P$, where $P$ is a product neighborhood of $\partial$, then $g \in \mathcal{D}^{r}(M)$.

Proof. It suffices to show that any $g \in G$ with $\operatorname{supp}(g) \subset \operatorname{Int}(P)$ is isotopic to the identity. Take a sequence $\lambda_{n}>0, n=0,1, \ldots$, tending increasingly to 1 . We may arrange so that $\left.g\right|_{\partial \times\left[0, \lambda_{0}\right]}=$ id.

Let an isotopy $f_{t}$ in $G$ be as in Def. 2.5(3). For $t \in(0,1]$ define

$$
g_{t}=f_{\frac{1-t}{t}} \circ g \circ f_{\frac{1-t}{t}}^{-1} \text {. }
$$


Then $g_{1}=g$ and $g_{t}$ extends smoothly onto $[0,1] \times P$ so that $g_{0}=\mathrm{id}$.

It follows from the assumption on $M$ and Proposition 2.8 that there is a compact set $C \subset M$ such that if $f \in \operatorname{Diff}^{r}(M)$ and $f \mid C=$ id then $f \in \mathcal{D}^{r}(M)$. Clearly, this is not true for all open manifolds with finite number of ends.

Lemma 2.9. If $G$ satisfies Definitions 2.1 and 2.5, then any $g \in G(P)$, where $P$ is a product neighborhood of $\partial$, can be written as a product of two commutators of elements of $G(P)$.

Proof. We may assume that $g \in G(\operatorname{Int}(P))$. Choose as in Def. 2.1 a sequence $0<a_{1}<\bar{a}_{1}<\bar{b}_{1}<b_{1}<a_{2}<\ldots<a_{n}<\bar{a}_{n}<\bar{b}_{n}<b_{n}<\ldots<1$ and $h \in G(P)$ such that conditions (2)-(5) in Def. 2.1 are fulfilled.Put $\bar{h}=h^{-1} g$, that is $g=h \bar{h}$. Then $\operatorname{supp}(\bar{h})$ is in $\left(0, \bar{a}_{1}\right) \cup \bigcup_{n=1}^{\infty}\left(\bar{b}_{n}, \bar{a}_{n+1}\right)$, and $\bar{h}=g$ on $\left[0, a_{1}\right] \cup \bigcup_{n=1}^{\infty}\left[b_{n}, a_{n+1}\right]$. It is easily observed that $\bar{h}$ also fulfills (2)-(5) in Def. 2.1. It suffices to show that $h$ is a commutator of elements in $G(\operatorname{Int}(P)$ ) (in the same way it is true for $\bar{h}$ ).

Choose arbitrarily $\lambda_{0} \in\left(0, a_{1}\right)$ and $\lambda_{n} \in\left(b_{n}, a_{n+1}\right)$ for $n=1,2, \ldots$ In view of Def. 2.5(3) there exists an isotopy $[0, \infty) \ni t \mapsto f_{t} \in G$ supported in $\partial_{G} \times(0,1)$, such that $f_{0}=$ id and $f_{j}\left(P_{n}\right)=P_{n+j}$ for $j=1,2, \ldots$ and for $n=$ $0,1,2, \ldots$, where $P_{n}=\partial_{G} \times\left[\lambda_{n}, 1\right)$ for $n=0,1, \ldots$ Now define $\tilde{h} \in G(\operatorname{Int}(P))$ as follows. Set $\tilde{h}=h$ on $\partial_{G} \times\left[0, \lambda_{1}\right)$, and $\tilde{h}=h\left(f_{1} h f_{1}^{-1}\right) \ldots\left(f_{n} h f_{n}^{-1}\right)$ on $\partial_{G(M)} \times\left[0, \lambda_{n+1}\right)$ for $n=1,2 \ldots$ Here $f_{n}=\left(f_{1}\right)^{n}$. Then $\left.\tilde{h}\right|_{\partial_{G} \times\left[0, \lambda_{n}\right)}$ is a consistent family of functions, and $\tilde{h}=\left.\bigcup_{n=1}^{\infty} \tilde{h}\right|_{\partial_{G} \times\left[0, \lambda_{n}\right)}$ is a local diffeomorphism. It is easily checked that $\tilde{h}$ is a bijection. Due to Def. 2.5(2) $\tilde{h} \in G(\operatorname{Int}(P))$.

By definition we have the equality $\tilde{h}=h f_{1} \tilde{h} f_{1}^{-1}$. It follows that $h=$ $\tilde{h} f_{1} \tilde{h}^{-1} f_{1}^{-1}$, as claimed.

Remark 2.10. It is necessary to use a decomposition $g=h \bar{h}$ in the above proof. In fact, we can proceed as above to define $\tilde{h}$ directly from $g$ (instead from $h$ ), but we cannot ensure that the resulting $\tilde{h}$ is surjective. On the other hand, if we would try to define $\tilde{h}$ by using $\tilde{g}_{n}=\left(f_{n} g f_{n}^{-1}\right) \ldots\left(f_{1} g f_{1}^{-1}\right) g$ on $\partial_{G(M)} \times\left[0, \lambda_{n+1}\right)$ then the family $\tilde{g}_{n}$ is inconsistent and we cannot glue-up $\tilde{g}_{n}$.

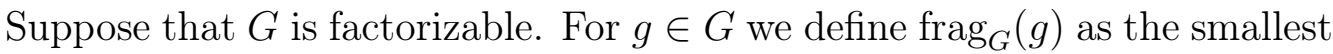
$N$ such that there are a family of balls $\left\{B_{\alpha}\right\}$, a product neighborhood $P$ and and a decomposition (2.1). Then frag $_{G}$ is a conjugation-invariant norm on $G$, called the fragmentation norm. In fact, since $G \subset \mathcal{D}^{r}(M)$, any $g \in G$ does not change the ends of $M$ so that it takes (by conjugation) any decomposition in the form (2.1) into another decomposition in the same form.

Define $\operatorname{fragd}_{G}:=\sup _{g \in G} \operatorname{frag}_{G}(g)$, the diameter of $G$ in $\operatorname{frag}_{G}$. Consequently, frag $_{G}$ is bounded iff $\operatorname{fragd}_{G}<\infty$. 
If $g \in G_{c}$ then, as usual ([5]), we define $\operatorname{Frag}_{G_{c}}(g)$ to be the smallest $N$ such that $g=g_{1} \ldots g_{N}$ with $g_{i} \in G\left(B_{i}\right), B_{i}$ being balls, $i=1, \ldots, N$, and $\operatorname{Fragd}_{G_{c}}:=\sup _{g \in G_{c}} \operatorname{Frag}_{G_{c}}(g)$.

Proposition 2.11. For all $J \subset K$ we have

$$
\operatorname{fragd}_{G}=\operatorname{fragd}_{G_{J}}=\operatorname{fragd}_{G_{c}}=\operatorname{Fragd}_{G_{c}} .
$$

Proof. If $g \in G_{c}$ then $\operatorname{Frag}_{G_{c}}(g) \geq \operatorname{frag}_{G_{c}}(g)$ as any fragmentation of $g$ supported in balls is of the form (2.1). On the other hand, if $g=g_{0} g_{1} \ldots g_{N^{\prime}}$ with $N^{\prime}<N=\operatorname{Frag}_{G_{c}}(g)$ is of the form (2.1), then $g_{0}^{-1} g \in G_{c}$ and $\operatorname{Frag}_{G_{c}}\left(g_{0}^{-1} g\right) \leq$ $N^{\prime}$. Hence fragd $_{G_{c}}=\operatorname{Fragd}_{G_{c}}$.

Next, let $g=g_{0} g_{1} \ldots g_{N} \in G$ and let $N=\operatorname{frag}_{G}(g)=\operatorname{fragd}_{G}$. Take $h=$ $g_{1} \ldots g_{N} \in G_{c}$. Then $\operatorname{frag}_{G_{c}}(h) \leq \operatorname{Frag}_{G_{c}}(h) \leq N$. But if $\operatorname{frag}_{G_{c}}(h)<N$ then $\operatorname{frag}_{G}(g)<N$, a contradiction. Therefore, $\operatorname{frag}_{G_{c}}(h)=\operatorname{frag}_{G}(g)$. It follows that $\operatorname{fragd}_{G}=\operatorname{fragd}_{G_{c}}$, since for $g \in G_{c}$ we have trivially $\operatorname{frag}_{G}(g)=\operatorname{frag}_{G_{c}}(g)$. Analogous statements are true for $G_{J}$ instead of $G_{c}$.

For any perfect group $G$ denote by $\operatorname{cld}_{G}$ the commutator length diameter of $G$, i.e. $\operatorname{cld}_{G}:=\sup _{g \in G} \operatorname{cl}_{G}(g)$. Then $G$ is uniformly perfect iff $\operatorname{cld}_{G}<\infty$.

Summing-up the above facts we have the following generalization of Theorem 1.1.

Theorem 2.12. Assume that $G \subset \operatorname{Diff}^{r}(M)$ satisfies Definitions 2.1 and 2.5. Then $G$ and $G_{J}(J \subset K)$ are perfect groups. Moreover, if there is a positive integer $r$ such that $\operatorname{cld}_{G(B)} \leq r$ for all balls $B$ and the fragmentation norm frag $_{G}$ of $G$ is bounded, then $G$ and $G_{J}$ are uniformly perfect and we have the inequalities

$$
\operatorname{cld}_{G} \leq r \operatorname{fragd}_{G}+2, \quad \operatorname{cld}_{G_{J}} \leq r \operatorname{fragd}_{G}+2 .
$$

Observe that Theorem 2.12 is not true for $\operatorname{Diff}_{J}^{r}(M) \cap \mathcal{D}^{r}(M)$ whenever $J$ is nonempty. In fact, for $J \subset K$, one has

$$
\left[\operatorname{Diff}_{J}^{r}(M) \cap \mathcal{D}^{r}(M), \operatorname{Diff}_{J}^{r}(M) \cap \mathcal{D}^{r}(M)\right]=\mathcal{D}_{J}^{r}(M),
$$

unless $J=K$ and $r=\operatorname{dim}(M)+1$, thanks to McDuff [16].

We will need some algebraic tools which mimic classical tricks for homeomorphism groups (see, e.g., [2]). A subgroup $H$ of $G$ is called strongly $m$ displaceable if there is $f \in G$ such that the subgroups $\mathrm{H}, \mathrm{fHf}^{-1}, \ldots, f^{m} \mathrm{Hf}^{-m}$ pairwise commute. Then we say that $f m$-displaces $H$. Fix a conjugationinvariant norm $\nu$ on $G$ and assume that $H \subset G$ is strongly $m$-displaceable. Then $e_{m}(H):=\inf \nu(f)$, where $f$ runs over the set of elements of $G$ that $m$-displaces $H$, is called the order $m$ displacement energy of $H$.

Now in view of Theorem 2.2 in [5] we have that given $G$ equipped with a conjugation-invariant norm $\nu$ and given $H \subset G$, a strongly $m$-displaceable 
subgroup of $G$, for any $h \in[H, H]$ with $\mathrm{cl}_{H}(h)=m$ one has

$$
\nu(h) \leq 14 e_{m}(H)
$$

and

$$
\mathrm{cl}_{G}(h) \leq 2 .
$$

If $m=1$, i.e. $h$ is a commutator of elements of $H$, then

$$
\nu(h) \leq 4 e_{1}(H) .
$$

In particular, if there exists $g \in G$ that $m$-displaces $H$ for every $m \geq 1$ the inequality (2.3) yields for all $h \in[H, H]$ that

$$
\nu(h) \leq 14 \nu(g) \text {. }
$$

A group $G \subset \mathcal{D}^{r}(M)$ is said to be locally moving if for any ball $B \subset M$ and any $x \in B$ there is $f \in G(B)$ with $f(x) \neq x$. Next, $G$ acts transitively inclusively on $M$ if for every balls $U$ and $V$ there is $f \in G$ with $f(U) \subset V$. It is clear that $\mathcal{D}^{r}(M)$ is locally moving and acts transitive inclusively on the basis of all balls.

Proposition 2.13. Assume that $G$ is locally moving and $G$ acts transitively inclusively on $M$. Then 0 is not an accumulation point in the set of values of any conjugation-invariant norm on $G$.

Proof. First observe the following fact:

(*) For any ball $U$ there are non-commuting $f_{1}, f_{2} \in G(U)$.

Indeed, take $x \in U$ and $f_{1} \in G(U)$ such that $f_{1}(x) \neq x$. Next choose $V \subset U$ with $x \in V$ and $f_{1}(x) \notin V$. Let $f_{2} \in G(V)$ such that $f_{2}(x) \neq x$. It follows that $f_{2}\left(f_{1}(x)\right)=f_{1}(x) \neq f_{1}\left(f_{2}(x)\right)$.

Fix $U$ and $f_{1}, f_{2}$ as in $(*)$. Let $\nu$ be a conjugation-invariant norm such that for any $\epsilon>0$ there is $g \in G$ with $0<\nu(g)<\epsilon$. As $g \neq$ id it follows the existence of a ball $B$ with $g(B) \cap B=\emptyset$. Since $G$ acts transitively inclusively there is $h \in G$ with $h(U) \subset B$. Therefore $h^{-1} g h(U) \cap U=\emptyset$. It follows from (2.5) that

$$
\nu\left(\left[f_{1}, f_{2}\right]\right) \leq 4 \nu\left(h^{-1} g h\right)=4 \nu(g)<4 \epsilon,
$$

a contradiction.

According to the terminology in [5] a group $G$ is called meagre if it is bounded and discrete. The latter means that 0 is not an accumulation point in the set of values of any conjugation-invariant norm on $G$.

Definition 2.14. (c.f. [5]) A smooth connected open manifold $M$ (with $M=$ $\operatorname{Int}(\bar{M})$, where $\bar{M}$ is a compact manifold) is called portable if it admits a complete vector field $X$ and a compact set $C \subset M$, called a core of $M$, such that the following conditions are satisfied: 
(1) for any compact set $K \subset M$ there is $t>0$ such that $\mathrm{Fl}_{t}^{X}(K) \subset C$ (here $\mathrm{Fl}_{t}^{X}$ is the flow of $X$ );

(2) there is $f \in \mathcal{D}_{c}^{r}(M)$ such that $f(C) \cap C=\emptyset$.

More generally, a connected open manifold $M$ of class $C^{r}, r=0,1, \ldots, \infty$, satisfies ( $\sqcup)$-property if there are disjoint open subsets $U, V$ of $M$ such that there is $f \in \mathcal{D}_{c}^{r}(M)$ with the closure of $f(U \cup V)$ contained in $V$, and such that for every $g_{1}, \ldots, g_{l} \in \mathcal{D}_{c}^{r}(M)$ there is $h \in \mathcal{D}_{c}^{r}(M)$ satisfying

$$
h\left(\bigcup_{i=1}^{l} \operatorname{supp}\left(g_{i}\right)\right) \subset U .
$$

It is immediate that any portable manifold satisfies the $(\sqcup)$-property.

Proposition 2.15. If $U, V$ are open disjoint subsets of $M$ such that there is $f \in \mathcal{D}^{r}(M)$ with $\overline{f(U \cup V)} \subset V$ then $f$ m-displaces $\mathcal{D}^{r}(U)$ for all $m \geq 1$.

Indeed, this follows from the relation $f^{m}(U) \subset f^{m-1}(V) \backslash f^{m}(V)$ for all $m \geq 1$.

The class of portable manifolds comprises the euclidean spaces $\mathbb{R}^{n}$, the manifolds of the form $M \times \mathbb{R}^{n}$, or the manifolds admitting an exhausting Morse function with finite numbers of critical points such that all the indices are less that $\frac{1}{2} \operatorname{dim} M$. In particular, every three-dimensional handlebody is a portable manifold.

It will be useful a more general notion concerning homeomorphism groups rather than manifolds.

Definition 2.16. A group $G \subset \mathcal{D}^{r}(M)$ on a manifold $M$ being the interior of a compact manifold is said to satisfy $(\mathcal{E})$-property if the following conditions hold:

(1) $G$ acts transitively inclusively on $M$.

(2) There are a ball $B$, an open subset $U \subset M$ disjoint with $B$ and $f \in G_{c}$ such that the closure of $f(U \cup B)$ is contained in $U$.

(3) If $j \in J_{G}$ and $P$ is a product neighborhood of $\partial$, then for any sequence in $(0,1)$, tending to 1 , of the form

$$
0<a_{1}<b_{1}<a_{2}<b_{2}<\ldots<a_{n}<b_{n}<\ldots<1
$$

there are $f_{1}, f_{2} \in G\left(\operatorname{Int}\left(P^{(j)}\right)\right)$ such that for $i=1,2, \ldots$ one has

$$
\begin{gathered}
f_{1}\left(\partial_{j} \times\left(\left[a_{2 i-1}, b_{2 i-1}\right] \cup\left[a_{2 i}, b_{2 i}\right]\right)\right) \subset \partial_{j} \times\left(a_{2 i}, b_{2 i}\right), \\
f_{2}\left(\partial_{j} \times\left(\left[a_{2 i}, b_{2 i}\right] \cup\left[a_{2 i+1}, b_{2 i+1}\right]\right)\right) \subset \partial_{j} \times\left(a_{2 i+1}, b_{2 i+1}\right) .
\end{gathered}
$$

Moreover, if we have another sequence tending to 1

$$
0<\tilde{a}_{1}<\tilde{b}_{1}<\tilde{a}_{2}<\tilde{b}_{2}<\ldots<\tilde{a}_{n}<\tilde{b}_{n}<\ldots<1
$$


then there is an element of $G\left(\operatorname{Int}\left(P^{(j)}\right)\right)$ of the form id $\times \varphi$, where $\varphi$ : $[0,1) \rightarrow[0,1)$ is a diffeomorphism, with $\varphi\left(a_{i}\right)=\tilde{a}_{i}$ and $\varphi\left(b_{i}\right)=\tilde{b}_{i}$ for $i=1,2, \ldots$.

Proposition 2.17. The groups $\mathcal{D}_{J}^{r}(M)(J \subset K)$ satisfy the $(\mathcal{E})$-property (Def. 2.16).

The proof is obvious.

Theorem 2.18. Let $J \subset K$. Suppose that $G \subset \operatorname{Diff}^{r}(M)$ satisfies Definitions 2.1, 2.5 and 2.16. Then the following conditions are equivalent:

(1) the norm frag $_{G}$ is bounded;

(2) $G$ is bounded;

(3) $G_{J}$ is bounded.

If $G$ is also locally moving then the above conditions are equivalent to the meagerness of $G$, or of $G_{J}$.

Proof. As frag $_{G}$ is a conjugation-invariant norm the implication $(2) \Rightarrow(1)$ is trivial. To show $(1) \Rightarrow(2)$ suppose that frag $_{G}$ is bounded. For any $g \in G$ we have a decomposition $g=g_{0} g_{1} \cdots g_{N}$ specified in Def. 2.1 with $N$ bounded. In particular, there is a family of balls $\left\{B_{\alpha}\right\}_{\alpha \in A}$ and a product neighborhood $P$ of $\partial$ such that $g_{0} \in G(P)$ and $g_{j} \in G\left(B_{\alpha(j)}\right)$ for $j=1, \ldots, N$.

Moreover, for $g_{0} \in G(P)$ there is a sequence, converging to 1 , of the form

$$
0<a_{1}<\bar{a}_{1}<\bar{b}_{1}<b_{1}<a_{2}<\ldots<a_{n}<\bar{a}_{n}<\bar{b}_{n}<b_{n}<\ldots<1
$$

and $h_{1}, h_{2} \in G(P)$ such that $h_{1}=g$ on $\bigcup_{n=1}^{\infty} \partial \times\left[\bar{a}_{2 n-1}, \bar{b}_{2 n-1}\right], \operatorname{supp}\left(h_{1}\right) \subset$ $U_{1}:=\bigcup_{n=1}^{\infty} \partial \times\left(a_{2 n-1}, b_{2 n-1}\right), h_{2}=g$ on $\bigcup_{n=1}^{\infty} \partial \times\left[\bar{a}_{2 n}, \bar{b}_{2 n}\right]$, and $\operatorname{supp}\left(h_{2}\right) \subset$ $U_{2}:=\bigcup_{n=1}^{\infty} \partial \times\left(a_{2 n}, b_{2 n}\right)$. Now, applying the reasoning from the proof of Lemma 2.9 for $h=h_{1} h_{2}$, it can be checked that $g_{0}$ can be written as $g_{0}=$ $h_{1} h_{2} h_{3} h_{4}$, where $h_{3}=g$ on $\bigcup_{n=1}^{\infty} \partial \times\left[b_{2 n-1}, a_{2 n}\right], \operatorname{supp}\left(h_{3}\right) \subset U_{3}:=\bigcup_{n=1}^{\infty} \partial \times$ $\left(\bar{b}_{2 n-1}, \bar{a}_{2 n}\right), h_{4}=g$ on $\bigcup_{n=0}^{\infty} \partial \times\left[b_{2 n}, a_{2 n+1}\right]$, and $\operatorname{supp}\left(h_{4}\right) \subset U_{4}:=\bigcup_{n=0}^{\infty} \partial \times$ $\left(\bar{b}_{2 n}, \bar{a}_{2 n+1}\right)$, for some $0<\bar{b}_{0}<b_{0}<a_{1}$. Furthermore, $h_{j}$ satisfy the corresponding conditions (2)-(5) in Def. 2.1.

It follows from Def. 2.16(2) and Proposition 2.15 (applied to $G$ ) the existence of a ball $B$ and $f \in G_{c}$ such that $\left\{f^{m}(B)\right\}_{m=0}^{\infty}$ is a pairwise disjoint family (here $f^{0}=\mathrm{id}$ ). In view of Def. 2.16(1) there are $h_{\alpha(j)} \in G_{c}$ such that $h_{\alpha(j)}\left(B_{\alpha(j)}\right) \subset B$ for $j=1, \ldots, N$. It follows that $\left\{h_{\alpha(j)}^{-1} f^{m} h_{\alpha(j)}\left(B_{\alpha(j)}\right)\right\}_{m=0}^{\infty}$ is a pairwise disjoint family. Consequently, $h_{\alpha(j)}^{-1} f h_{\alpha(j)} m$-displaces $G\left(B_{\alpha(j)}\right)$ for all $m \geq 1$ and for $j=1, \ldots, N$.

Likewise, in view of Def. 2.16(3) and Proposition 2.15 we have the existence of $f_{j} \in G$ with $\operatorname{supp}\left(f_{j}\right) \subset \partial_{G} \times(0,1)$ such that $G\left(U_{j}\right)$ is $m$-displaceable by $f_{j}$ for $j=1,2,3,4$ and for all $m \geq 1$. 
Let $\nu$ be a conjugation-invariant norm on $G$. In view of (2.6) and the invariance of $\nu$ we have

$$
\begin{aligned}
\nu(g) & \leq \nu\left(h_{1}\right)+\cdots+\nu\left(h_{4}\right)+\nu\left(g_{1}\right)+\cdots+\nu\left(g_{N}\right) \\
& \leq 14\left(\nu\left(f_{1}\right)+\cdots \nu\left(f_{4}\right)+N \nu(f)\right) .
\end{aligned}
$$

Although the sets $U_{1}, \ldots, U_{4}$ depend on $g_{0}$ (and on $g$ ), thanks to the second assertion of Def. 2.16(3) and the invariance of $\nu$, the norms $\nu\left(f_{j}\right)$ are independent of $g$. It follows that $\nu(g)$ is bounded, as required.

In view of Proposition 2.11 we have that (1) is equivalent to (3) in the same way. The second assertion is a consequence of Proposition 2.13.

Corollary 2.19. Let $\mathcal{D}(M)=\mathcal{D}^{r}(M), r=0,1, \ldots, \infty, r \neq \operatorname{dim}(M)+1$. The following conditions are equivalent:

(1) the norm $\operatorname{frag}_{\mathcal{D}(M)}$ is bounded;

(2) $\mathcal{D}(M)$ is bounded;

(3) $\mathcal{D}_{J}(M)$ is bounded;

(4) $\mathcal{D}_{c}(M)$ is bounded;

(5) $\mathcal{D}(M)$ is meagre;

(6) $\mathcal{D}_{J}(M)$ is meagre;

(7) $\mathcal{D}_{c}(M)$ is meagre.

It is a consequence of Propositions 2.7, 2.13 and 2.17, and of Theorem 2.18.

Proof of Theorem 1.2 and Corollary 1.3. Let $M$ satisfy the ( $\sqcup)$-property. In view of Propositions 2.7 and 2.17, the group $\mathcal{D}^{r}(M)$ fulfills Definitions 2.1, 2.5 and 2.16 if $r \neq \operatorname{dim}(M)+1$. By (2.7), Proposition 2.15 and (2.6) it is easy to check that $\mathcal{D}_{c}^{r}(M)$ is bounded. Therefore, by Corollary 2.19 the groups $\mathcal{D}^{r}(M)$ and $\mathcal{D}_{J}^{r}(M)$ are bounded. In particular, these groups are uniformly perfect. The inequality $\operatorname{cld}_{\mathcal{D}_{J}^{r}(M)} \leq 4$ follows from Proposition 2.7, Lemma 2.9 , and the fact that $\operatorname{cld}_{\mathcal{D}_{c}^{r}(M)} \leq 2$ (Theorem 1.18 with Remark 3.2 in [5]).

\section{EXAMPLES}

First recall the following basic fact, c.f. [5].

Proposition 3.1. Let $G$ be any group. If $H_{1}(G)$ is infinite then $G$ is unbounded.

The first example reveals the significance of the property that $G(M)$ is "determined on compact subsets" (Def. 2.5(2)).

Example 3.2. Let $N$ (resp. $S$ ) be the north (resp. south) pole of $\mathbb{S}^{m}$, and let $p_{N}: \mathbb{S}^{m} \backslash\{N\} \rightarrow \mathbb{R}^{m}$ (resp. $p_{S}: \mathbb{S}^{m} \backslash\{S\} \rightarrow \mathbb{R}^{m}$ ) be the corresponding stereographic projection. By $\mathcal{D}_{c}^{\infty}\left(\mathbb{R}^{m}, 0\right)$ we denote the identity component of compactly supported $C^{\infty}$-diffeomorphisms of $\mathbb{R}^{m}$ fixing the origin $0 \in \mathbb{R}^{m}$. 
Define $G\left(\mathbb{S}^{m}\right)=p_{N}^{-1} \mathcal{D}_{c}^{\infty}\left(\mathbb{R}^{m}, 0\right) p_{N}$. Then $G\left(\mathbb{S}^{m}\right) \subset \mathcal{D}^{\infty}\left(\mathbb{S}^{m}\right)$ and any element of $G\left(\mathbb{S}^{m}\right)$ preserves $S$. Next, define $G\left(\mathbb{R}^{m}\right)=p_{S} G\left(\mathbb{S}^{m}\right) p_{S}^{-1}$. It is easily checked that $G\left(\mathbb{R}^{m}\right) \cong \mathcal{D}_{c}^{\infty}\left(\mathbb{R}^{m}, 0\right)$ and $G\left(\mathbb{R}^{m}\right)$ fulfils Def. 2.1 and (1), (3) in Def. 2.5. Notice that Def. 2.5(2) does not hold. In fact, it suffices to take any $g \in \mathcal{D}^{\infty}\left(\mathbb{S}^{m} \backslash S\right)$ which is not smoothly extendable on $\mathbb{S}^{m}$, and $f=p_{S} g p_{S}^{-1}$. Then $f \notin G\left(\mathbb{R}^{m}\right)$ but $f$ fulfills the assumption of Def. 2.5(2). Note that $G\left(\mathbb{R}^{m}\right)$ does not satisfy (the last statement) of Def. 2.16(3).

On the other hand, since $H_{1}\left(G\left(\mathbb{R}^{m}\right)\right)=H_{1}\left(\mathcal{D}_{c}^{\infty}\left(\mathbb{R}^{m}, 0\right)\right)=\mathbb{R}$ by a theorem of K. Fukui in [9, the group $G\left(\mathbb{R}^{m}\right)$ is unbounded by Proposition 3.1.

Similarly, let $\mathcal{D}_{l, c}^{\infty}\left(\mathbb{R}^{m}, 0\right)$ be the identity component of compactly supported $C^{\infty}$-diffeomorphisms of $\mathbb{R}^{m}$ fixing the origin and $l$-tangent to the identity at the origin $(l \geq 1)$. In the same manner as above we define a group $G_{l}\left(\mathbb{R}^{m}\right)$ which satisfies Def. 2.1 and Def. 2.5, (1) and (3), but not Def. 2.5(2) and not Def. 2.16(3). In view of $\left[9\right.$ we have $H_{1}\left(G_{l}\left(\mathbb{R}^{m}\right)\right)=H_{1}\left(\mathcal{D}_{l, c}^{\infty}\left(\mathbb{R}^{m}, 0\right)\right)=\mathbb{R}^{l+1}$ so that the group $G_{l}\left(\mathbb{R}^{n}\right)$ is unbounded due to Proposition 3.1.

A conjugation-invariant norm $\nu$ on a group $G$ is stably bounded if the limit $\lim _{n \rightarrow \infty} \frac{\nu\left(g^{n}\right)}{n}=0$ for any $g \in G$. It is clear that any bounded $\nu$ is also stably bounded. Next, a map $\varphi: G \rightarrow \mathbb{R}$ is called a quasi-morphism if there is $K>0$ such that $|\varphi(g h)-\varphi(g)-\varphi(h)| \leq K$ for any $g, h \in G$. A deep theorem of C. Bavard [4] states that the commutator length $\mathrm{cl}_{G}$ is stably unbounded if and only if there exists a non-trivial (i.e. not being a morphism) homogeneous quasi-morphism on $G$.

Example 3.3. Let $\mathcal{H}(\mathbb{R})$ be the group of all homeomorphisms $h$ of $\mathbb{R}$ which verifies $h(x+1)=h(x)+1$ for all $x \in \mathbb{R}$. In view of 8$] h \in \mathcal{H}(\mathbb{R})$ is a product of $p$ commutators if and only if

$$
\inf _{x \in \mathbb{R}}(h(x)-x)<2 p-1 \text { and } \sup _{x \in \mathbb{R}}(h(x)-x)>1-2 p .
$$

The group $\mathcal{H}(\mathbb{R})$ admits a quasi-morphism $\tau$, called the translation number, given for $h \in \mathcal{H}(\mathbb{R})$ by the formula

$$
\tau(h)=\lim _{n \rightarrow \infty} \frac{h^{n}(x)-x}{n},
$$

which is independent of $x \in \mathbb{R}$ thanks to (3.1), c.f. [4]. By the theorem of Bavard it follows that the commutator length is stably unbounded on the commutator subgroup $[\mathcal{H}(\mathbb{R}), \mathcal{H}(\mathbb{R})]$ so that the commutator length on $[\mathcal{H}(\mathbb{R}), \mathcal{H}(\mathbb{R})]$ is unbounded. In view of Proposition 1.4 in [5], the group $\mathcal{H}(\mathbb{R})$ is itself unbounded.

This is encoded in the fact that Def. 2.1 is not fulfilled by $\mathcal{H}(\mathbb{R})$, while Def. 2.5 holds. Indeed, Def. 2.5(1) is satisfied due to 14 and Def. 2.5(2) is trivial. To show Def. $2.5(3)$ we identify $[0,1)$ with $[0, \infty)$ and define $[0, \infty) \ni t \mapsto$ $f_{t} \in \mathcal{H}(\mathbb{R})$ to be a continuous curve of translations. 
On the other hand, according to classical results of A. Denjoy [6] $\tau$ restricted to the subgroup in $\mathcal{H}(\mathbb{R})$ of strictly increasing smooth diffeomorphisms is trivial so that the theorem of Bavard does not apply in this case. Notice that $\mathcal{D}^{\infty}(\mathbb{S})$, which is isomorphic to the subgroup of strictly increasing smooth diffeomorphisms in $\mathcal{H}(\mathbb{R})$, is bounded due to Theorem 1.11 in [5].

Considering some subgroups of the symplectomorphism group or of the volume preserving diffeomorphism group may be a source of examples of automorphism groups of open manifold that fulfill Def. 2.1 and Def. 2.5, (1) and (2), but not Def. 2.5(3). For instance, let $G\left(\mathbb{R}^{2 m}\right)$ be the kernel of Calabi homomorphism of $\mathbb{R}^{2 m}$ equipped with the standard symplectic form. It is well-known that $G\left(\mathbb{R}^{2 m}\right)$ is a simple group by a classical result of A. Banyaga, c.f. 3]. Recently, D. Kotschick in [11] proved that this group is also stably bounded.

Observe that $G\left(\mathbb{R}^{2 m}\right)$ is a compactly supported group. It is not difficult to extend $G\left(\mathbb{R}^{2 m}\right)$ to a symplectomorphism group $\hat{G}\left(\mathbb{R}^{2 m}\right)$ with no restriction on support by making use of Def. 2.1. Then $\hat{G}\left(\mathbb{R}^{2 m}\right)$ satisfies Def. 2.1 and Def. 2.5, (1) and (2), but not Def. 2.5(3). The reason is that the symplectic volume is a symplectic invariant. Consequently, Kotschick's criterion for stable boundedness of the commutator length (Theorem 2.3 in [11]) cannot be extended to groups with no restriction on support.

\section{REFERENCES}

[1] K. Abe, K. Fukui, Commutators of $C^{\infty}$-diffeomorphisms preserving a submanifold, $J$. Math. Soc. Japan 61(2009), 427-436.

[2] R.D.Anderson, On homeomorphisms as products of a given homeomorphism and its inverse, Topology of 3-manifolds, ed. M. Fort (Prentice-Hall, 1961), 231-237.

[3] A. Banyaga, The structure of classical diffeomorphism groups, Mathematics and its Applications, 400, Kluwer Academic Publishers Group, Dordrecht, 1997.

[4] C. Bavard, Longeur stables des commutateurs, Enseign. Math. 37 (1991), 109-150.

[5] D. Burago, S. Ivanov and L. Polterovich, Conjugation invariant norms on groups of geometric origin, Advanced Studies in Pures Math. 52, Groups of Diffeomorphisms (2008), 221-250.

[6] A. Denjoy, Sur les courbes definies par les équations différentielles à la surface du tore, J. Math. Pures Appl. (9), 11(1932), 333-375.

[7] R. D. Edwards, R. C. Kirby, Deformations of spaces of imbeddings, Ann. Math. 93 (1971), 63-88

[8] D. Eisenbud, U. Hirsch and W. Neumann, Transverse foliations of Seifert bundles and self homeomorphisms of the circle, Comment. Math. Helv. 56 (1981), 639-660.

[9] K. Fukui, Homologies of the group $\operatorname{Diff}^{\infty}\left(\mathbb{R}^{n}, 0\right)$ and its subgroups, J. Math. Kyoto Univ. 20 (1980), 475-487

[10] M. W. Hirsch, Differential Topology, Graduate Texts in Mathemetics 33, Springer 1976.

[11] D. Kotschick, Stable length on stable groups, Advanced Studies in Pures Math. 52, Groups of Diffeomorphisms (2008), 401-413. 
[12] J. Lech, T. Rybicki, Groups of $C^{r, s}$-diffeomorphisms related to a foliation, Banach Center Publ. 76(2007), 437-450.

[13] W. Ling, Translations on $M \times \mathbb{R}$, Amer. Math. Soc. Proc. Symp. Pure Math. 32, 2 (1978), 167-180.

[14] J. N. Mather, The vanishing of the homology of certain groups of homeomorphisms, Topology 10(1971), 297-298.

[15] J. N. Mather, Commutators of diffeomorphisms, Comment. Math. Helv. I 49 (1974), 512-528; II 50 (1975), 33-40; III 60 (1985), 122-124.

[16] D. McDuff, The lattice of normal subgroups of the group of diffeomorphisms or homeomorphisms of an open manifold, J. London Math. Soc. (2), 18(1978), 353-364.

[17] T. Rybicki, The identity component of the leaf preserving diffeomorphism group is perfect, Monatsh. Math. 120 (1995), 289-305

[18] T. Rybicki, Commutators of diffeomorphisms of a manifold with boundary, Annales Pol. Math. 68(1998), 199-210.

[19] T. Rybicki, On the group of diffeomorphisms preserving a submanifold, Demonstratio Math. 31(1998), 103-110.

[20] P. A. Schweitzer, Normal subgroups of diffeomorphism and homeomorphism groups of $\mathbb{R}^{n}$ and other open manifolds, preprint (2009).

[21] W. Thurston, Foliations and groups of diffeomorphisms, Bull. Amer. Math. Soc. 80 (1974), 304-307.

[22] T. Tsuboi, On the group of foliation preserving diffeomorphisms, (ed. P. Walczak et al.) Foliations 2005, World scientific, Singapore (2006), 411-430.

[23] T. Tsuboi, On the uniform perfectness of diffeomorphism groups, Advanced Studies in Pures Math. 52, Groups of Diffeomorphisms (2008), 505-524.

Faculty of Applied Mathematics, AGH University of Science and Technology, al. Mickiewicza 30, 30-059 Kraków, Poland

E-mail address: tomasz@uci.agh.edu.pl 